\title{
SU UN CASO DI POLMONITE DA
} PNEUMOCYSTIS CARINII

Fabbrizi V., Sisino L.; Seca P.

Laboratorio Patologia Clinica;

Ospedale Civile "G. Mazzini" Teramo - Settore Microbiologia

Introduzione. Pneumocystis carinii è un organismo unicellulare in parte assimilato ai funghi per alcune caratteristiche genetiche ed in parte ai protozoi specie per la sua suscettibilità a famaci generalmente attivi su questi microrganismi. Il parassita viene trasmesso per via aerea e rimane silente a livello alveolare per manifestare la sua patogenicità solo in soggetti immunodepressi nei quali causa una polmonite interstiziale.

La diagnosi di certezza si basa sulla dimostrazione di $P$. 
carinii su liquido di broncolavaggio o espettorato indotto. Il presente lavoro illustra il caso di una giovane donna di etnia orientale, sieropositiva, giunta al reparto di Infettivologia del nostro ospedale con dispnea ingravescente e cianosi.

Sono stati eseguiti sulla paziente esami di chimica clinica ed emocromocitometrici e, dopo consenso informato il test per l'HIV. Attestata la sieropositività della paziente, si sono eseguiti broncolavaggi per la ricerca di $P$. carinii.

Metodi. i numerosi broncolavaggi eseguiti sono stati trattati con una metodica atta a rilevare la presenza di cisti e trofozoiti di $P$. carini con il metodo della fluorescenza diretta.

Risultati. Tutti i broncolavaggi eseguiti sulla paziente prima del trattamento antibiotico mirato e il miglioramento delle condizioni cliniche, mostravano positività alla fluorescenza diretta per numerose cisti e trofozoiti di $P$. carini.

Conclusioni. Contestualmente al miglioramento delle condizioni cliniche della paziente in seguito all'adeguata somministrazione di terapia antibiotica, il numero di cisti di $P$. cari$n i$ rilevate per campo microscopico è diminuito fino alla negativizzazione dei broncolavaggi.

La paziente è stata dimessa e seguita per alcuni mesi dal reparto di Infettivologia. Attualmente se ne sono perse le tracce. 